\title{
Use of screencasting to facilitate engineering course delivery
}

Paul R. Hampson

School of Computing, Science and Engineering, The University of Salford, Salford. UK Email: p.r.hampson@salford.ac.uk

\begin{abstract}
The use of technology to enhance learning opportunities is increasing and interventions such as screencasting can have benefits in providing the student with a flexible learning resource. Within this study, a screencast tutorial was produced for engineering students to teach the process of modelling laminated materials within the commercial finite element software, ANSYS. The effectiveness of the screencast was assessed using an anonymous online questionnaire and small focus group discussion. Overall, feedback from students was positive and found that the majority of students preferred to use a dual learning approach by using the screencast along with an accompanying PDF step-by-step guide. It was also evident from both questionnaire and focus group responses that the instructor-learner interaction was still regarded by the students as an important aspect of the course delivery; especially for teaching advanced concepts when using finite element software.
\end{abstract}

Keywords screencast; technology enhanced learning; accessibility; evaluation; engineering 


\section{Introduction}

Screencasting is a form of online training that utilises video demonstration typically with accompanying audio commentary ${ }^{1}$ that is beginning to replace or supplement some of the more traditional forms of delivering training and lecture material. ${ }^{2}$

Increasing amounts of literature is now becoming available for screencasting which is most notably due to the advancements that have been made in data connection speeds provided by modern computer networks, and the availability of various platforms (e.g. computers and smartphones) that are able to directly access this media rich format thus providing asynchronous learning opportunities. ${ }^{3}$ Students are also spending greater amounts of time online and using the internet to access study materials. ${ }^{4,5}$

Specific to the discipline of engineering courses, screencasting has been used to supplement student learning within a material science and engineering course at the University of Michigan ${ }^{6}$ in which additional course material was presented including homework solutions, quizzes and mini-lectures. Their study found that although most students benefited from the screencasts, the effectiveness could be improved if both staff and students were able to increase their utilisation of the new teaching medium. An updated paper on this study also notes that screencasting can have benefits for accommodating differing learning styles since written, audio and visual elements can all be contained within one presentation, and improved student final grade marks can also be achieved if the screencast is aligned with the course learning objectives. ${ }^{7}$ 
Screencasts have also been utilised to teach mathematic concepts at James Cook University in which the authors used Camtasia software to create a library of video lectures. ${ }^{5}$ The overall conclusion from the paper states that the use of screencast was positively received both by staff and students, although from the lecturer's perspective, a significant amount of time was required to produce the online material. The time required to produce a video tutorial which included captioning was also discussed in the work of Garvett and Gill, ${ }^{8}$ where they state it was a lengthy process which required support from another member of staff. Despite this, once created, the screencast can minimise the need for the lecturer to give repetitive explanations and the material can remain valid for several years. ${ }^{5}$

The threat that screencasts present to the loss of jobs within the education sector is also briefly discussed by Mullamphy et al., ${ }^{5}$ and although there's room for further debate, they confirm that at present there is no evidence to support this. It's further noted that indeed, students would not like to see lectures being fully replaced by online content since concerns have been expressed about the impact of the loss of instructor-learner interaction, ${ }^{9}$ and Mullamphy et al. ${ }^{5}$ found in their study that students still required immediate interaction with the lecturer to help them understand difficult concepts that required deeper explanation. Despite this loss of interaction, a screencast will still be of great benefit and most students report that they find screencast helpful, independent of whether they found the lesson topic difficult or not. ${ }^{7}$ Concerns have been expressed in literature that the use of online lecture material may result in a drop in student attendance, ${ }^{10}$ although some studies have shown that this may not necessarily be the case. ${ }^{11}$ 
Screencasts also benefit those students who require flexibility in their studies since they can be viewed 'on demand' at any time ${ }^{12}$ and can be paused, rewound, and reviewed as many times as the student needs. ${ }^{5}$ The accessibility features contained within the software used to generate the screencast can also benefit students with disabilities. ${ }^{13,14}$

Whether screencasts are used to replace or supplement lecture material, the use of technology to enhance teaching is certainly increasing and perhaps the fact that most people now have access to home computers with reasonably high-speed internet connections and smartphones that can access web media content makes this trend inevitable. Many modern students also need part-time jobs to help fund their education which in turn means they may be unable to physically attend some lectures; this will require more flexible learning patterns ${ }^{5}$ which can be supported with Technology Enhanced Learning (TEL) interventions such as screencasting. 


\section{Methodology}

\section{Description of the problem}

Students on undergraduate and postgraduate engineering courses at the University of Salford are required to complete a Finite Element Analysis (FEA) module that comprises a series of theoretical lectures and practical computer software based workshops. Within the computer workshops, the students are introduced to a commercial FEA software package called ANSYS in which they complete several tutorial exercises and assessments that predominantly use metallic material properties to teach the use of the software. In its current form, the tutorial examples are provided as printed step-by-step tutorial examples. This is a successful approach since programming metallic (isotropic) material properties into the software is a relatively straight forward task, thus allowing the student to focus on the more challenging aspects of using the software for the first time. Despite this, some students may in the future be required to model laminated materials; typically advanced composite (orthotropic) materials with various layer configurations which requires a deeper understanding of the software; particularly important for many final year undergraduate and postgraduate projects for which there are no tutorials. The ability to understand how to implement composite material properties into FEA software will also be extremely important for those students who may later gain future employment in structural stress analysis offices.

Since there are no formal exercises within the current module which introduce composite material modelling, students would typically approach the author on an ad-hoc basis when they 
needed to learn these techniques; particularly for individual project work which requires these skills. This approach proved to be very time consuming and involved a lot of repetition to different students.

The aim of this study is therefore, to determine if screencast methods can form a teaching resource that will meet the needs of the students and staff, and to evaluate delivery and design.

\section{Target audience}

The audience for the new screencast material developed in this study consisted of 38 engineering final year undergraduate students in its first year of deployment, followed by 36 students in the second year of review.

The majority of students were male, home students, although the cohort still included a small but significant number of overseas candidates. This meant consideration needed to be given to language abilities and educational cultural differences. Whatever background the student came from, it was expected that prior to studying engineering at the University of Salford, the candidate would have predominantly studied science and mathematical based disciplines and have a generally good technical understanding. Despite this, most students were unlikely to have encountered finite element methods or associated software previously in their prior studies so would initially have no theoretical understanding or practical application using specialist software. 


\section{TEL intervention}

By developing an online screencast tutorial and supporting documentation, it will allow students to be directed to the new material either as an extended tutorial session within formal teaching contact hours, or as self-directed study to be conducted in the students own time. This will alleviate the time constraints of providing ad-hoc teaching and also ensure that all students (not just those who conduct project work) are better prepared when faced with industrial applications of the software in their future careers.

The activity involves the use of an online video demonstration and accompanying step-by-step written guide to explain how to use ANSYS software to model and analyse laminated materials to help students develop important practical skills in the use of the software.

Firstly, a slideshow presentation was created that introduced and defined the engineering problem to be solved which was then incorporated as an introduction to an online video tutorial created with Camtasia screencasting software. This software was chosen after the author had experimented with alternate packages, and found that Camtasia produced very good video quality with reasonable file sizes. The Camtasia user interface included not only the recording facility, but also very powerful (and very easy to use) post-processing controls to edit the video. The final video included annotation and narration to provide further detail and describe options within the software. The screencast was a self-contained teaching resource in that the student had access to all of the information they needed to successfully complete the intended learning outcomes independently; without the need for additional staff support. The tutorial example was 
also produced as an accompanying step-by-step PDF document that detailed the techniques to use in the software.

\section{Cognitive loading considerations}

On the whole, most authors of screencasts agree that video tutorials should be kept as short as possible in order to retain the student's interest and concentration, ${ }^{1}$ with a value of 15 minutes or less being regarded as ideal. ${ }^{3}$ This is primarily due to the fact that a multimedia format can increase the cognitive load since much more information has to be processed by the student, e.g. graphics, text, and audio. ${ }^{4,15}$ Since this is the case, several authors recommend caution before using screencasts and ask the reader to consider if training could be provided in a more effective medium such as a step-by-step instructional PDF guide. ${ }^{1,4}$

The presentation of information can have the potential to cognitively overload the student and cause distractions to the learning process. ${ }^{16,17}$ Careful consideration was therefore given to the 'Split-attention principle' ${ }^{15,18}$ which effectively states that within a multimedia presentation, words should be provided as an audio commentary rather than on-screen text in order to maximise their effectiveness.

From a multimedia perspective, it's been suggested that a learner is capable of visual information processing and verbal information processing which can be utilised simultaneously to maximise the effectiveness of teaching material by allowing the student to generate two different mental models, thus permitting a much stronger chance of knowledge retention. ${ }^{15,17}$ 
For this teaching intervention study, the creation of an ANSYS software online video demonstration with annotation and narration already incorporated both of these aspects, however, consideration was given to using on-screen text sparingly; limited to only a single word or two that helped to highlight a particular feature of the software rather than provide a descriptive text that was better suited to audio delivery.

\section{Accessibility considerations}

Accessibility and equality needs to be considered for all of the student population and particularly for those with disabilities such as impaired hearing, blindness and physical

impairment. ${ }^{13,19}$ Accessibility legislation not only makes this a legal requirement, but also ensures that we provide the best learning opportunities for our students. The most common accessibility guidelines for general web authors are provided by the World Wide Web Consortium $^{20-22}$ who primarily split the approach into two sections: (i) Technical Accessibility and (ii) Usability. Without the former, the student would be unable to access the learning resource, and without the latter, the resource may not be easy to use or navigate. Although screencasts aren't explicitly covered within these guidelines, there are many recommendations that do apply. ${ }^{13}$

Specific to the multimedia area of screencasts, many publications report the process and methods for generating instructional videos but very few publications address their accessibility considerations. There are however two very good papers that do approach this aspect; Oud ${ }^{13}$ 
provides guidelines and techniques to develop accessible screencasts, and Wakimoto \& Soules ${ }^{14}$ provide a detailed comparison for three screencast creation products; Camtasia, Captive and VoiceThread with regard to their accessibility features.

A summary of the key considerations specific to screencasts as presented by $\mathrm{Oud}^{13}$ are given below:

- $\quad$ Provide alternate formats (e.g. text based tutorial)

- Allow keyboard access (e.g. can the video be controlled without a mouse?)

- Provide time control to adjust the rate of tutorial delivery. (e.g. pausing, rewind, and fastforward)

- Include voice narration

- Provide 'Alt' descriptive text that can be utilised by screen-reading software.

- Provide captions

For the ANSYS video tutorial created in this study, both voice narration and an alternate, detailed step-by-step instructional text was presented to the students. The accompanying PDF format of the tutorial could then either be used in conjunction with the video or as a stand-alone tutorial sheet if the student chose not to engage with the screencast.

\section{Supporting students using technology}


The student population is becoming increasingly "techno-savvy"5 and spending greater amounts of time online using multimedia environments. ${ }^{4,23}$ It was therefore anticipated that the final year engineering students (with two years prior use of computers on the degree programme) would have a good understanding of how to use computers and experience very few problems with the technology utilised for engaging with the screencast tutorial. The university also provides adequate computing facilities and additionally, most students have their own personal computers and smartphones that work equally as well.

Due to the potentially large file sizes associated with the developed screencast, it was decided that the best method of content delivery would be to upload the content onto YouTube and stream the videos by providing the students with direct weblinks. The advantages of YouTube are that it's one of the most widely used video sharing sites, ${ }^{24}$ most students are familiar with the facility, and aren't required to enter usernames and passwords in order to access it. This familiarity and ease of $u^{24}$ also helped to greatly reduce the number of problems associated with the technology.

Despite all of this, the author still endeavoured to support the students if they experience technical difficulty by providing contact details and, if necessary, giving the students the option of face-to-face help to resolve their technology problems. Smyth \& Mainka ${ }^{25}$ describe this form of support as 'Continuous facilitation'. However, due to the advantages stated above, it should be noted that once the new teaching material was introduced to the students, the author received no requests for further assistance. 


\section{Evaluation strategy}

The process of evaluating and assessing TEL interventions can be a challenging task. ${ }^{8}$ It is important to use a mixed method approach to gather both quantitative and qualitative feedback which can be typically collected using techniques such as surveys and focus groups.

"Use a variety of methods so that findings from one source can substantiate others.",26

In Gravett \& Gill's ${ }^{8}$ analysis of their online video tutorial, they report that an anonymous comment form did not generate much feedback and that "Student time constraints and reluctance to critique staff and/or resources", 8 could perhaps be considered as possible reason for low feedback volume. They therefore intended to use focus groups to supplement the data. Surveys were also used in Mullamphy et al's ${ }^{5}$ investigation into the effectiveness of mathematics screencasts. Their voluntary survey consisted of 9 multichoice questions and 3 open questions which resulted in a response from $33 \%$ of the student cohort.

Phil Race, ${ }^{27}$ suggests that most questionnaires contain a mix of closed and open-ended style questions. Closed questions offer more controlled responses whereas open-ended allow the candidate more freedom to answer in their own words. ${ }^{28,29}$

For the evaluation of this TEL intervention, both questionnaire (anonymous online survey) and focus group were used. Once the online tutorial had been used by the students, they were invited to complete a feedback questionnaire that was developed to clearly target specific questions that 
enabled accurate assessment of the success of the chosen multimedia format and also generated information in order to make appropriate improvements for future screencasts. For the $1^{\text {st }}$ cohort, 17 out of 38 candidates (45\%) responded to the survey, followed by 19 out of $36(53 \%)$ for the

$2^{\text {nd }}$ cohort. A focus group was then conducted that contained randomly selected candidates ${ }^{30}$ and took the form of a semi-structured discussion that allowed for fluidity to ensure the candidates were unrestricted in their responses. ${ }^{25}$ Therefore, by having a combination of both quantitative and qualitative methods, ${ }^{31}$ it was possible to capture more in-depth feedback that otherwise would have been difficult to extract from a questionnaire alone. ${ }^{26}$ For both years of screencast review, 10 candidates were selected randomly and invited by email to join the focus group discussion; 5 candidates positively responded to the request in the $1^{\text {st }}$ cohort, and 4 candidates in the $2^{\text {nd }}$ cohort. 


\section{Results \& Discussion}

This section presents an evaluation and critical reflection on the successfulness of the intervention by reviewing the feedback information obtained via questionnaire and focus group. Consideration is also given to accessibility, future developments and the opportunities and constraints identified from the study.

\section{Questionnaire results}

An anonymous survey was conducted online using Surveymonkey which consisted of 7 short questions compiled from a mix of multiple choice and open response style questions. The questions were designed to extract information the author felt would judge how well the initial screencast had been produced, plus also offer insight into where improvements could be made. Open-ended questions were also deliberately included to allow the student freedom to discuss things that hadn't been thought of, and to offer a more general response.

To reduce/eliminate any rogue responses, the students were emailed with a direct web link for the survey. The web link was otherwise unpublished. Once the survey period was completed, the author reviewed the data and took advantage of Surveymonkey's IP address reporting feature to check for unique entries (the author could not identify individual candidates with this information thus, preserving anonymity). 
Fundamentally, the questionnaire was designed to give feedback about the screencast in terms of its usefulness for teaching and also practical aspects of the video such as narration pace.

A summary of the responses from both cohorts is presented below:

Question 1: How useful was the screencast format for teaching ANSYS software?

Possible responses were of the Likert-type scale with options of; very poor, poor, neutral, good, and very good. From the $1^{\text {st }}$ year of survey data, $47.1 \%$ responded 'good' and $52.9 \%$ 'very good', and for the $2^{\text {nd }}$ year; $5.3 \%$ 'neutral', 36.8\% 'good', and 57.9\% 'very good'. It was encouraging to see that all of the responses were positive and students found the ANSYS video tutorial useful. The more examples a student has access to increases the ease at which they can conduct their own studies and projects. Highlighting key features within the software can save the student many hours of trying to locate the right options and settings themselves.

Question 2: Which format (e.g. video / written document) did you prefer to use?

Current ANSYS workshops begin with an initial 'live' online demonstration conducted with the use of a computer connected to a projector, followed by the student progressing at their own pace through step-by-step PDF guides. From Figure 1, it is interesting to note that the PDF format has not been made completely redundant by the screencast since most of students used a 'dual' format approach to their learning. This reinforces the comment by Meehan \& Hyland ${ }^{1}$ that for more complex applications of screencasts, a PDF document can still have benefits. 
Question 3: Did narration help you to understand the tutorial?

All of the responses from both cohorts to this question agreed that for the example demonstrated in the screencast, narration helped with the understanding of the software. It was perhaps inevitable that narration would only assist since the descriptive audio explained exactly what was happening at each step of the analysis.

Question 4: Was the pace of the video too slow, just right, or too fast?

From Figure 2, the pace of the video tutorial appears to have been acceptable for the majority of users. For the rest who stated the pace was too slow for them (and the one candidate who reported it was too fast), they always have the option to utilise the playback features available within most media players to either increase/decrease the speed of the video as required, or to 'skip' to different sections.

Question 5: Did the video tutorial achieve its objectives?

As stated in the introductory section of the video tutorial, there were three aims which where to understand:

- Basic modelling of laminated materials (orthotropic properties). 
- How to use ESHAPE command to view 3D representation of Real Constant data assigned to $2 \mathrm{D}$ models.

- Create LAYER STACKING plots to verify layer material and direction information.

Possible responses were of the Likert-type scale with options of; strongly disagree, disagree, neither disagree nor agree, agree, and strongly agree. From the survey response, $52.9 \%$ 'agreed' and $47.1 \%$ 'strongly agreed' in the $1^{\text {st }}$ years cohort, followed by $57.9 \%$ 'agreed' and $42.1 \%$ 'strongly agreed' for the $2^{\text {nd }}$ cohort.

Question 6: What suggestions do you have for improving the tutorial?

- Comment 1: "Tips on how to obtain material properties (e.g. shear modulus), possibly a reference to a book you feel can instruct students on this"

- Comment 2: "It would have been better if the video was continuous (not in two parts)"

- Comment 3: "Very good as a basic modelling of composites tutorial, however, would be useful to include more advanced information on material properties of composites as well as issues (limitations) when using FEA."

- Comment 4: "For an absolute beginner it may be worth explaining why the boundary conditions at the edge of the plate are applied to 2 planes, not 3."

Comments 1 and 3 are valid questions but were actually outside of the scope of the 15 minute ANSYS screencast which clearly stated learning outcomes that were specific software modelling processes rather than discussion of the material properties which is the concern of other modules 
on the undergraduate programme. This however, perhaps demonstrates the need for instructorlearner interaction that can address these issues as they arise.

Comment 2 is also valid, but in this instance the author was restricted to a maximum video duration of 15 minutes which forced the production of a separate 4 minute 'Introduction' and a 15 minute 'Tutorial' section.

Question 7: If you would like to provide any additional comments, please include the here.

- Comment 1: "Good additional tips on viewing the layup * Identifying real/visualised (ESHAPE) * Possibly some information on plate analysis' boundary conditions for the newbies (UX, UZ fixed, but why? - few even know of poisson's ratio) * Maybe limitations of this tool WRT to real-world/simulation models (source of high error due to manufacture style/accuracy, assume no loss of energy \& perfect bending, etc)"

- Comment 2: "This tutorial will be useful for my final year project."

A lot of the points made in comment 1 are very good ideas and although not directly addressed in the current training video, they would probably make useful topics for future screencasts, indeed, selecting the correct boundary conditions could be a presentation in its own right. Some of the points would be better addressed by recording a 'mini-lecture' rather than demonstrating within the actual ANSYS software.

\section{Focus Group}


For the $1^{\text {st }}$ cohort, a focus group was conducted with 5 students who had all used the new training video. The key aims of the focus group were to find out more detailed information specific to the effectiveness of the intervention in which the discussions were broadly based on the following questions:

- "Does the screencast provide a useful supplement to the practical ANSYS workshops?"

- "Does the screencast substitute the need for face-to-face teaching?"

Although the focus group had some structure, the format of the meeting was deliberately kept flexible to ensure the students could comment freely and perhaps raise areas of discussion that had not originally been considered. During the discussion, comments were recorded and some of the key responses/themes are presented below:

- Comment 1: "Where did the Shear Modulus values come from?"

- Comment 2: "Can I refer the video to my friends outside of the university?"

- Comment 3: "The pace was a bit slow - I clicked forward through some sections."

- Comment 4: "I watched the video on my phone but couldn't get the survey to work."

- Comment 5: "Although the screencast was helpful, I still want to be able to speak to the lecturer."

Comments 1, 3 and 5 reinforce some of the comments extracted from the online survey. As before, comment 1 and 5 highlight the fact that perhaps an instructor-learner interaction would 
be beneficial. For comment 1, the scope of the tutorial was not to teach material properties; the aims and objectives were clearly stated in the introductory material and this was not one of them, but despite this, the student has been inspired to ask the question.

Although comment 3 appears to be negative and describes the pace being slow, it's interesting to note that the candidate "clicked forward through some sections", this comment most usefully demonstrates one of the key advantages of using screencasts in that for students who are 'quicker' at understanding concepts are not restricted to keeping pace with participants who require more time. The fact that this student had the facility to progress through the material at a faster pace must have actually been quite a time-saving feature.

Most interesting are comments 2 and 4. Firstly, the student who requested permission to email the web links to his friends studying overseas at other universities stated that his reasons for doing so was because there weren’t many (if any) similar training videos for modelling laminated materials, and he knew of several people who would find the screencast beneficial. Indeed, within the scope of this study, the screencast was already available globally since it had been uploaded unrestricted to YouTube. However, this does in itself raise an important question; "Should the screencast be restricted to 'fee paying' students enrolled at the university or should they be available freely on the global stage?".

Would a student paying tuition fees to a university be happy that the teaching material they're paying for could just have easily have been accessed, without cost, from a simple internet search? Perhaps a compromise could be achieved where a select few tutorials could be placed on 
public access servers such as YouTube since this would help to raise the teaching profile of the university and help attract new students, but also ensure that some material is exclusive to feepaying students by placing the material on university streaming servers where access is provided only to students enrolled on the module.

Comment 4 , provided a useful insight into some of the challenges in using relatively new technology. Prior to inviting students to trial the screencast and complete the online survey, the author tested the weblinks using his own computer and smartphone to confirm that everything worked well. This process also included re-uploading the videos to YouTube several times to observe the effect of different settings and resolutions to ensure the best quality would be available to the students.

What hadn't been considered was incompatibility with different smartphone systems. This particular student was able to view the YouTube videos on his smartphone but unable to access the online questionnaire. The reasons for this are still unclear; since they both simply require website access then it would have been expected that both would have been equally accessible. However, advanced smartphones are still relatively new devices with different operating systems and standards so perhaps, as with any new technology, it was not surprising that issues like this occur from time-to-time. In future, a key discussion topic (for the face-to-face focus group) would be "What equipment did you use to access the training material and did you encounter any problems?”. 
The $2^{\text {nd }}$ cohort focus group was conducted with 4 students. Discussions and comments typically reiterated the $1^{\text {st }}$ cohort with the main exception being the following comment:

- Comment 1: "I watched the video at home but couldn't practice along with it since I don't have ANSYS software on my laptop."

The comment above highlights the fact that not everybody will have access to the software offcampus. However, the university provides several computing suites within the Engineering department which have ANSYS installed and can be accessed by the students outside of their timetabled classes if they wish to spend further time with the software.

\section{YouTube Statistics}

As of $15^{\text {th }}$ June 2015, there had been 296 views of the 'Introduction' and 2183 views of the main training video. The latter also featured a comment left by a YouTube user:

- "Thank you for your in-depth video in solving this problem. I was having issues solving the same problem from the verification manual but you have really helped me out. Thanks!" 


\section{Conclusion}

The screencast provided the students with a learning resource that could be accessed at any time to help facilitate flexible learning patterns and also addressed the time constrains of the author in providing ad-hoc training.

Fundamentally, the majority of students preferred to use a dual learning format by using the screencast along with an accompanying PDF step-by-step guide. It was also evident from both questionnaire and focus group responses that the instructor-learner interaction was still regarded by the students as an important aspect of the course delivery; especially for advanced concepts when using finite element software. Throughout the study, the students reported they had no problems with the technological knowledge required to access the learning resource, and the only exception was the isolated smartphone web access problem for completing the online survey.

Ad-hoc training for individual students would typically take between 30 to 60 minutes dependent of their understanding of the use of ANSYS finite element software. This was found to be significantly reduced by using the screencast and associated written material which took approximately half a day to produce. The advantage of the screencast also meant the students weren't restricted to the time spent with the author but were able to review the taught material as often as they wished, and to learn the new material at their own pace. The continued availability of the screencast and supporting PDF document also means the author can continue to take advantage of the resource by referring subsequent cohorts to the material in future years, with only the need for moderate refinements to keep the material up-to-date and relevant. 


\section{Suggestions for future improvements}

Within the Camtasia software used to produce the screencast training video, there were two additional facilities that could have been utilised to enhance the presentation. The first, would have been to include captions that would have improved the accessibility of the video. This was omitted from the current tutorial primarily due to time constraints and, after a review of relevant student support plans for the target audience, on this occasion there were no students that would require the use of captions. Since the author had a good knowledge of the ANSYS software used in the screencast demonstration, a script hadn't been written for the narration which lead to a more spontaneous approach being taken that made the narration sound more natural and also saved time during the production phase. However, for future screencast, it would be useful to write a script so that elements of it could be 'copy-and-pasted' into the Camtasia caption format and synchronised with the video narration.

The second feature in Camtasia that was deliberately omitted was the inclusion of a 'picture-inpicture' display of the author providing narration whilst demonstrating the software. It was considered that this would have no additional teaching benefit and if anything, actually detract from it by providing a distraction from the main focus of the screencast. Indeed, in the study

conducted by Mullamphy et al, ${ }^{5}$ they report that out of a group of mathematics and IT lecturers only one of them made use of this facility, and they question whether its use helped to make the presentation appear more personal or just caused unnecessary distraction. 
Finally, a general, non-software specific improvement, would have been to include a summary at the end of the presentation to help reinforce key learning concepts. ${ }^{4}$ 


\section{References}

1. Meehan D and Hyland J. Video killed the 'PDF' star: taking information resource guides online. SCONUL Focus, 47 (Winter) 2009; pp.23-27.

2. McGarr O. A review of podcasting in higher education: Its influence on the traditional lecture. Australasian Journal of Educational Technology 2009; vol. 25, no. 3: 309-321.

3. Yee K and Hargis J. Screencasts. Turkish Online Journal of Distance Education 2010; vol. 11, no. 1: 9-12.

4. Oud J. Guidelines for effective online instruction using multimedia screencasts. Reference Services Review. 2009; vol. 37, no. 2: 64-177.

5. Mullamphy DF, Higgins PJ, Belward SR. and Ward LM. To screencast or not to screencast. ANZIAM Journal, vol. 51 (EMAC2009) 2010 pp.446-460.

6. Pinder-Grover T, Millunchick JM and Bierwert C. Work in progress - Using screencasts to enhance student learning in a large lecture material science and engineering course. 38th ASEE/IEEE Frontiers in Education Conference, October 22-25 2008, Saratoga Springs, New York. 
7. Pinder-Grover T, Millunchick JM, Bierwert C and Shuller L. Leveraging screencasts to strategically clarify unclear materials science concepts. Paper presented at the American Society of Engineering Education Annual Conference and Exposition, Austin, Tx, June 14-17, 2009.

8. Gravett K and Gill C. Using online video to promote database searching skills: the creation of a virtual tutorial for Health and Social Care students. Journal of information literacy 2010; vol. 4, no. 1: 66-71.

9. Rose K. Student perceptions of the use of instructor-made videos in online and face-to face classes. MERLOT Journal of Online Learning and Teaching 2009; vol. 5, no. 3: 487-495.

10. Kolowich S. Fans and fears of 'lecture capture'. Inside Higher Education, http://www.insidehighered.com/news/2009/11/09/capture (2009, accessed 20 May 2012).

11. Cramer K, Collins K, Snider D and Fawcett G. Virtual lecture hall for in-class and online sections: A comparison of utilization, perceptions and benefits. Journal of Research on Technology in Education, 2006; vol. 38, no. 4: 371-381.

12. Pinder-Grover T, Green KR and Millunchick JM. The efficacy of screencasts to address the diverse academic needs of students in a large lecture course. Advances in Engineering Education, 2011; vol. 2, no. 3: 1-28. 
13. Oud J. Improving screencast accessibility for people with disabilities: Guidelines and techniques. Internet Reference Services Quarterly 2011; vol. 16, no. 3: 129-144.

14. Wakimoto DK and Soules A. Evaluating accessibility features of tutorial creation software. Library Hi Tech 2011; vol. 29, no. 1: 122-136.

15. Mayer RE and Moreno R. A cognitive theory of multimedia learning: Implications for design principles. Paper presented at the CHI-98 Workshop on Hyped-Media to Hyper-Media 1998. Los Angeles.

16. Sweller J. Cognitive load theory, learning difficulty, and instructional design. Learning and Instruction, 1994; vol. 4, no. 4: 95-312.

17. Mayer RE. Multimedia learning: Are we asking the right questions? Educational Psychologist, 1997; vol. 32, no. 1: 1-19.

18. Chandler P and Sweller J. Cognitive load theory and the format of instruction. Cognition and Instruction, 1991; vol. 8, no. 4: 93-332.

19. Smyth K and Mainka C. Pedagogy and learning technology: a practical guide. Edinburgh Napier University Press. Unit 8: Issues in student support. http://staff.napier.ac.uk/services/academicdevelopment/PALT/Documents/epegagogy guide UNIT08.pdf (2010 accessed 30 April 2012). 
20. World Wide Web Consortium (W3C). Web content accessibility guidelines (WCAG) 2.0. http://www.w3.org/TR/WCAG20/ (2008 accessed 26 April 2012).

21. World Wide Web Consortium (W3C). Techniques and failures for Web Content Accessibility Guidelines (WCAG) 2.0. http://www.w3.org/TR/WCAG20-TECHS/ (2010a accessed 26 April 2012).

22. World Wide Web Consortium (W3C). Introduction to web accessibility. http://www.w3.org/WAl/intro/accessibility (2010b accessed 26 April 2012).

23. Lippincott JK. Net generation students and libraries. EDUCAUSE Review, 2005; vol. 40, no. 2: 56-66.

24. Snelson, C Web-based video in education: Possibilities and pitfalls. TCC 2008 Proceedings, pp.214-221.

25. Smyth K and Mainka C. Pedagogy and learning technology: a practical guide. Edinburgh Napier University Press. Unit 9: Evaluating technology-supported learning. http://staff.napier.ac.uk/services/academicdevelopment/PALT/Documents/epegagogy guide UNIT09.pdf (2010 accessed 30 April 2012). 
26. Learning Technology Dissemination Initiative. The Evaluation Cookbook. Scottish Higher Education Funding Council. http://www.icbl.hw.ac.uk/ltdi/cookbook/cookbook.pdf (1998 accessed 30 April 2012).

27. Race P. 2000 Tips for lecturers. London: Kogan Page, 1999.

28. Oppenheim AN. Questionnaire design, interviewing and attitude measurement. London: Pinter, 1992. pp.112-115.

29. Converse JM and Presser S. Survey questions: handcrafting the standardized questionnaire. London: Sage, 1986, p33.

30. Fowler FJ. Improving survey questions: design and evaluation. London: Sage, 1995, p.107.

31. Hall G. Project planning: Evaluation plan. JISC, http://www.jisc.ac.uk/fundingopportunities/projectmanagement/planning/evaluation.aspx (2012 accessed 24 January 2012). 


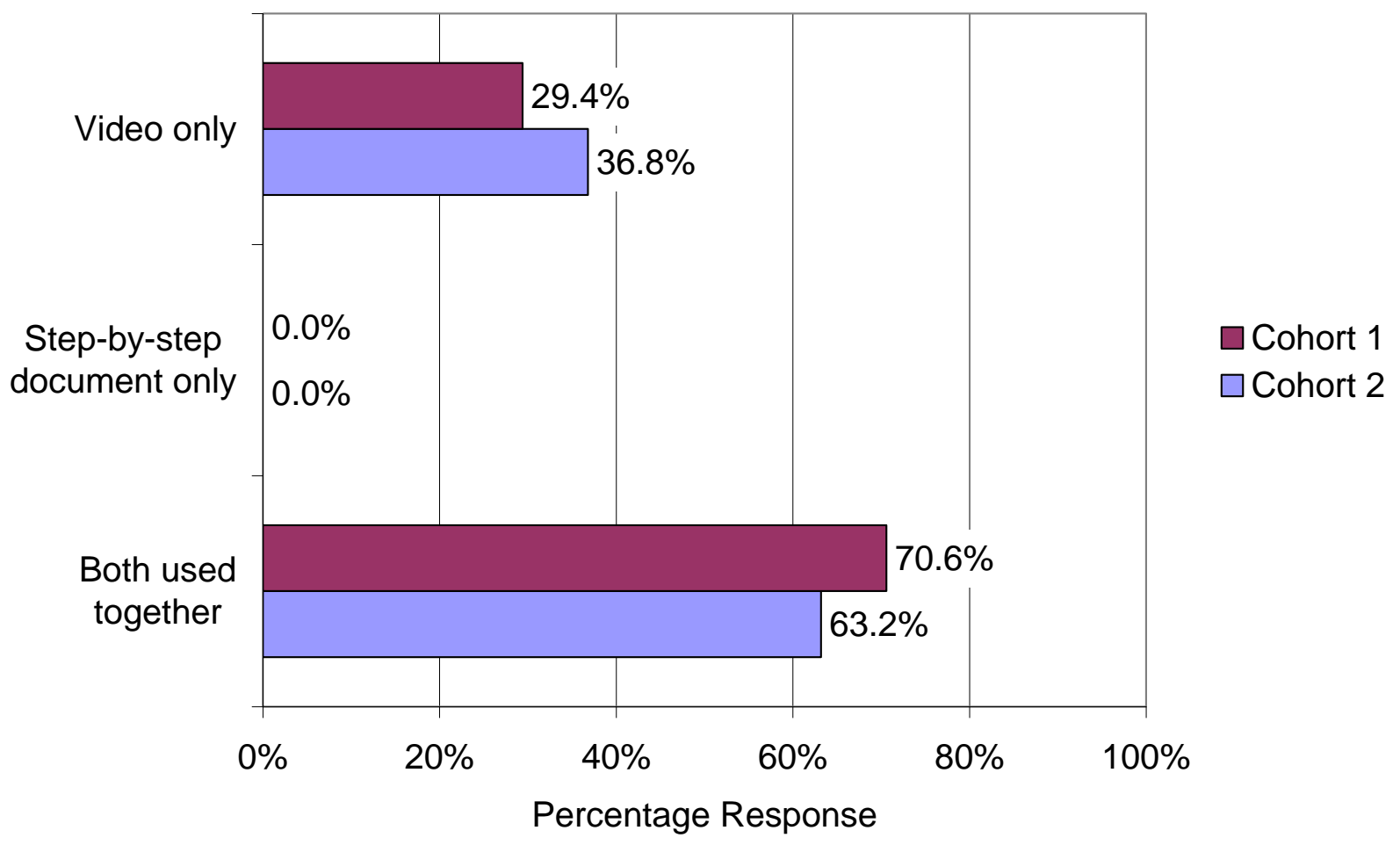

Figure 1: Format preference response (question 2) 


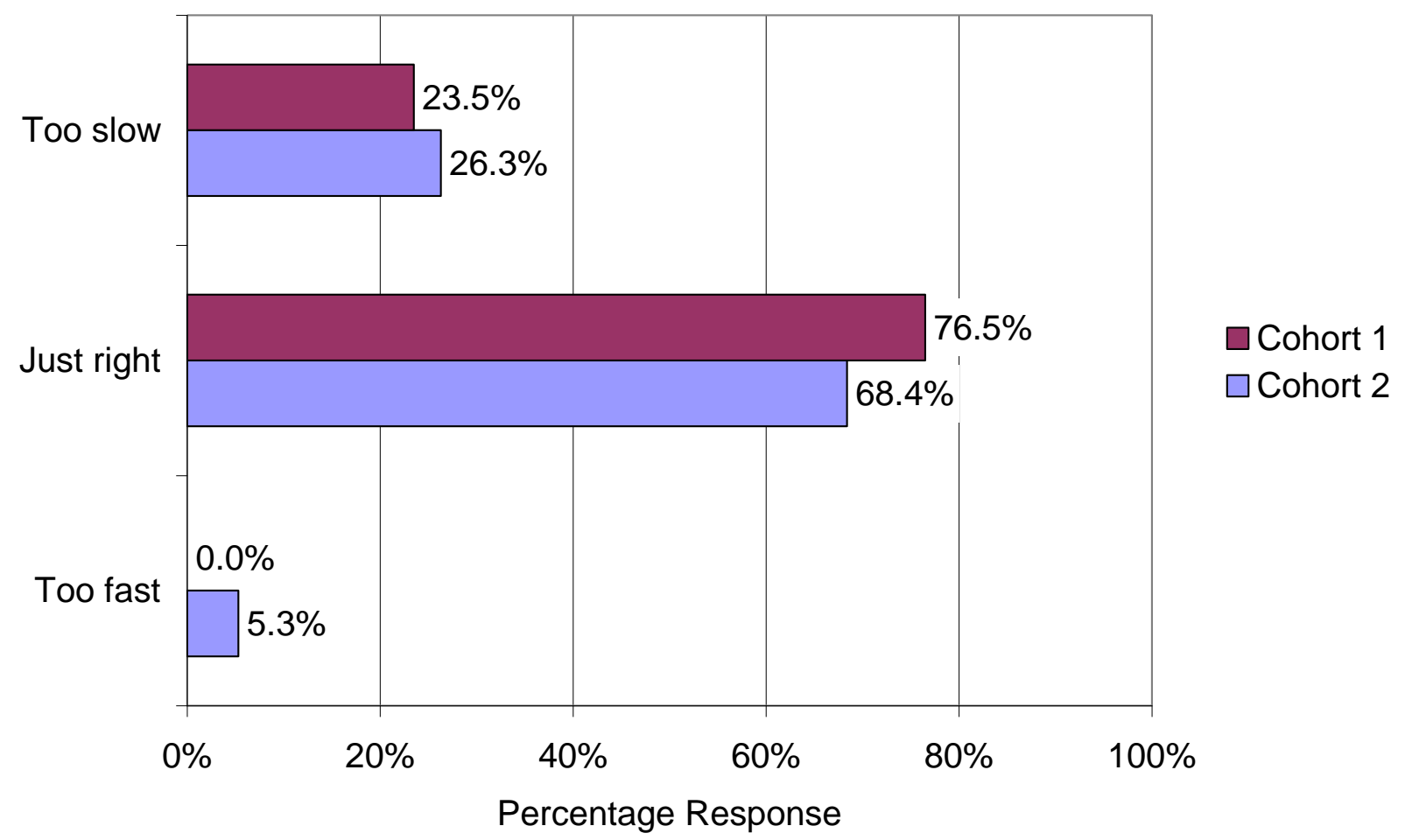

Figure 2: Pace of screencast response (question 4) 